\title{
Quantifying Lattice Distortions in Alloys
}

\author{
L.R. Owen ${ }^{a}$, N.G. Jones ${ }^{a, *}$ \\ ${ }^{a}$ Department of Materials Science and Metallurgy, University of Cambridge, CB3 OFS, UK
}

\section{ARTICLE INFO}

\section{Keywords:}

High entropy alloys

Local lattice strain

\begin{abstract}
A B S TRACT
Quantification of local lattice strain is essential for enhancing our understanding of structure-property relationships, particularly for high entropy alloys; but no common framework currently exists, with a number of possible quantifications in the literature. Here, local static and dynamic displacements are discussed, and different methods of describing their statistical dispersion presented. Similarly, the type of distribution measured, either offsite displacements or bond length, is considered alongside the different experimental and modelling techniques that can yield these distributions. This leads to a suggested numerical quantification for local lattice strain, to enable cross comparison between alloy systems using data obtained from different methodologies.
\end{abstract}

\section{Introduction}

Since the inception of the high-entropy alloy field there has been extensive debate around the four key principles defined by Yeh et al [1,2]. Of these, the highly distorted lattice remains the most poorly understood and least discussed [3, $4,5]$. It has been proposed that the existence of such highlydistorted lattices would dramatically increase the solid-solution strengthening properties of high-entropy alloys, compared with compositionally simpler materials [2]. However, despite a number of studies attributing physical property changes to this effect $[1,6,7,8]$, and attempts to rationalise this with physical models $[9,10,11,12]$, the fidelity of this principle has yet to be conclusively proven, mainly due to the lack of comparative experimental evidence between alloys with differing compositional complexity.

A number of studies have endeavoured to tackle this issue and explore whether the structures of such alloys can indeed be considered highly strained. Various experimental methods have been employed including diffraction [13, 14, 15], microscopy [8], total scattering [16, 17, 18, 19] and EXAFS [20], as well as theoretical ab initio calculations [20, 21, 22]. However, some confusion exists regarding the measurement of these off-site displacements and, even when viable data have been obtained, there is no consensus as to its numerical quantification. For example, a number of different formulations exist within the literature, confounding what is meant by 'local-lattice strain' and preventing easy comparison between different studies. Without a common quantification to enable comparison between data collected from different sources on different materials, it will be difficult for the community to conclusively establish the solid-solution strengthening component that results from local distortions, and whether indeed HEAs contain significantly higher levels of distortion.

The concept of strain is commonly understood in materials science. For example, when an object of length $L$ is placed under a normal force, it is observed to distort, with

\footnotetext{
*Corresponding author

@ lo250@cam.ac.uk (L.R. Owen); ngj22@cam.ac.uk (N.G. Jones)

ORCID(s): 0000-0003-2311-3908 (L.R. Owen)
}

the length becoming $L+\Delta L$. This leads to the standard definition of engineering strain, $\varepsilon_{\mathrm{Eng}}=\frac{\Delta L}{L}$. Many types of strain are defined, often separated into types depending on the length-scale over which they act [23], but in all cases the key point is that strain is a dimensionless quantity defined by the ratio of a change in a distance relative to a reference distance. Therefore, when considering the concept of a local lattice strain, two key questions need to be asked. First, what is the appropriate change in distance that we are interested in exploring? Second, what is the fair reference state for a comparison?

To answer these two questions, we will consider, compare and clarify some of the numerical expressions for local lattice strain currently used in the literature. By doing so, we will identify the most appropriate approach for quantification. Critically, this approach facilitates cross comparison between datasets from any system even when obtained using different methodologies, thus enabling the lattice distortion effect in HEAs to be investigated and compared.

\section{Distortion of the structure}

Consider a monatomic metal, where the atoms are modelled as 'hard-spheres'. It should be noted that this common approximation is really a description of the space in which the electrons can be found with a certain probability. At $0 \mathrm{~K}^{1}$ the arrangement is such that all of the atoms are touching (Fig. 1(a)(i)). As the atoms have no thermal energy, they remain stationary and hence their position at any time is coincident with their average position (white spots in Fig. 1(a)(ii)).

As the temperature is increased, the atoms begin to oscillate. This results in dynamic offsite displacements i.e. displacements, $u$, away from the average site, as a function of time. Consequently, the atoms now occupy a larger volume in space (shown by the dotted red lines), resulting in the thermal expansion of the structure Fig. 1(b)(i). It can be seen that the average positions of the atoms (red spots) have shifted from their previous location. Fig. 1(b)(ii) shows a plot of the positions of the atomic centres as a function of

\footnotetext{
${ }^{1}$ assuming no zero-point energy
} 
(a)

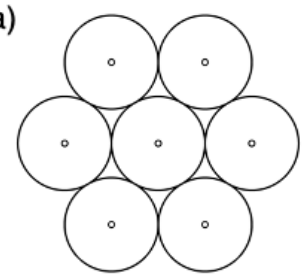

(i)
Monatomic, OK

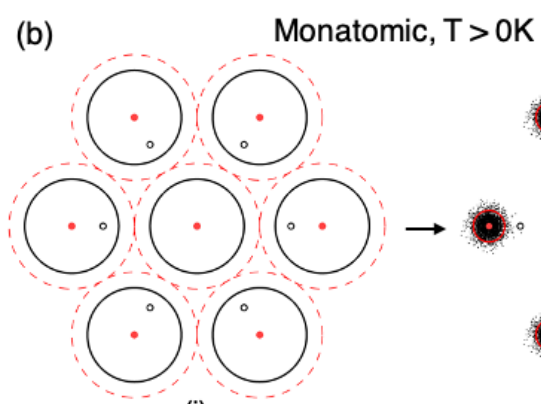

(i)

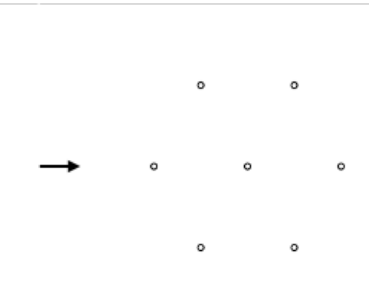

(ii)

(c)

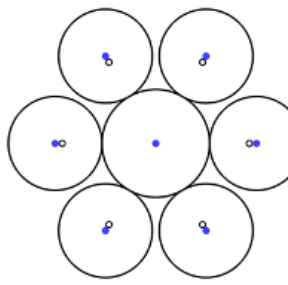

(i)

Alloy, OK

(d) Alloy, $\mathrm{T}>\mathrm{OK}$

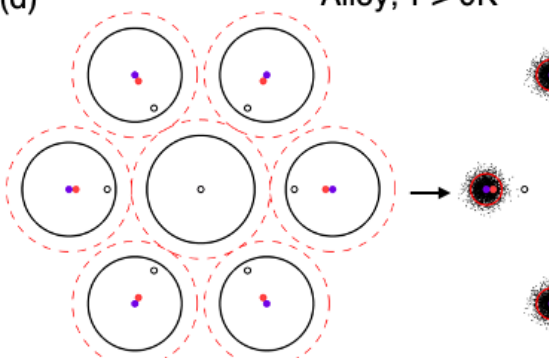

(i)

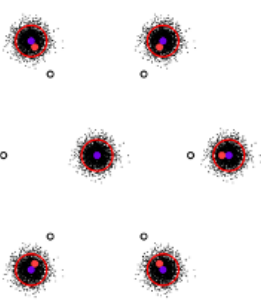

(ii)

Figure 1: Graphical representation of materials at different temperatures. (a) Monatomic structure at 0K, (b) Monatomic structure at $T>0 \mathrm{~K}$, (c) Substituted alloy structure at $0 \mathrm{~K}$, (d) Substituted alloy structure at $T>0 \mathrm{~K}$. (i) Hard sphere type model, (ii) the position of the atomic centres as a function of time. Black circles - hard sphere atoms, red dashed circle - shell in space occupied by an atom undergoing thermal oscillations. Black dots - position of atomic centres as a function of time. Spots indicate the average positions of the atomic centres: white - for monatomic at 0K, red - with thermal displacement, blue - with static displacement, purple - with both thermal and static displacements. Red circle - bounding region containing certain probability of atoms under dynamic displacements.

time (black dots). Similar to the comment made about the position of the electrons, the position of the atoms will form a statistical distribution rather than a precise value.

For a distribution, such as these positions, it is possible to calculate statistical parameters such as the standard deviation, $\sigma$, and the average $\mu$. If these positions are averaged over time, $\langle u\rangle_{t}$, they would yield the average atomic centres (red spots) i.e. the atoms undergo dynamic motion around these thermal centres. This distribution is often modelled by a thermal ellipsoid showing a region in which there is a certain probability of finding an atom at any time (red line). For metals, this thermal ellipsoid is typically isotropic (and Gaussian), as shown here, but this need not be the case. In Fig. 1, the radius of the sphere has been approximated by $2 \sigma$, which for a 3D Gaussian is $\approx 74 \%$.

In this case the change in the radius of the spherical shell occupied by the atom (black line to red dashed line) is the same as the radius of the thermal ellipsoid (red line). From this definition, a thermal strain could be described by considering the change in the radius of the sphere occupied by the thermally oscillating atom compared with that occupied by the non-oscillating atom at $0 \mathrm{~K}$ :

$$
\varepsilon_{u, \text { thermal }}=\frac{\sigma_{u, T}}{\bar{r}}
$$

where $\bar{r}$ is the average atomic radius, $\sigma_{u, T}$ is the standard deviation of the atomic positions (subscript $T$ indicates this displacement is due to thermal oscillations). For plotting thermal ellipsoids, many metrics could be used for the radii, e.g. $2 \sigma$, or the fullwidth at half maximum $(2 \sqrt{2 \ln 2} \sigma \approx$ $2.355 \sigma$ ), corresponding to different probabilities. However, these are merely linear scales, and so yield a multiple of the strain defined in the formulation above.

If a substitutional solid solution is considered, where host atoms are replaced by ones with a different atomic radii, an isotropic distortion field is produced, displacing neighbouring atoms away from their idealised sites, Fig. 1(c)(i). Whilst this will occur in all alloy systems, it has been suggested that the large mix of atoms of different sizes in a high entropy solid solution may have a particularly large high level of offsite displacement [2, 24]. These offsite displacements are static i.e. they do not vary as a function of time (see Fig. 1(c)(ii)). Hence, unlike dynamic displacements, they will be observed in the material at $0 \mathrm{~K}$.

These static offsite displacements, commonly referred to as local lattice strain, will only be apparent when comparing the actual atomic positions to the idealised atomic positions, and so require spatial averaging, $\langle u\rangle_{s}$. Spatial averaging is equivalent to condensing all the atoms in the structure down onto a single site and considering the displacements away from that site. Like the dynamic displacements, the strain field for most metals is generally isotropic.

Fig. 2(i) shows the spatial average of the thermal oscillations from Fig. 1(b). It is apparent that this yields the same result as the temporal average for the dynamic displacements (as would be expected). Fig. 2(ii) shows the spatial average of the static displacements shown in Fig. 1(c). The bounding line shows the $2 \sigma$ radius that, were there to be more atoms 


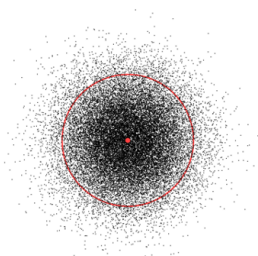

(i)

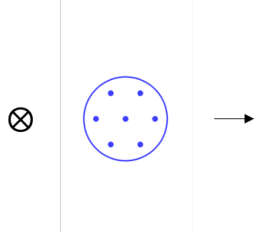

(ii)

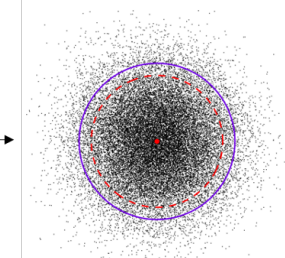

(iii)
Figure 2: Spatially averaged distribution of atomic positions. (i) Dynamic displacements, (ii) Static displacements, (iii) Static and dynamic displacements. Black dots - atomic positions. Circles - bounding region containing certain probability of atoms under: Blue - static displacements, Red - dynamic displacements, Purple - static and dynamic displacements

averaged, would contain the same probability of atomic centres as the bound of the thermal ellipsoid.

As with the thermal strain, the distribution of the atomic centres under static displacements, will define a statistical distribution. Hence, it is possible to describe a static strain in an analagous manner to a thermal strain:

$$
\varepsilon_{u, \text { static }}=\frac{\sigma_{u, S}}{\bar{r}}
$$

where $\sigma_{u, S}$ is the standard deviation of the static displacements away from the idealised location ${ }^{2}$.

The combined effect of static and dynamic displacements for an alloy at $T>0 \mathrm{~K}$ is shown in Fig. 1(d). It is apparent that, due to the static displacements, the thermal oscillations are taking place around sites (purple spots) that have shifted relative to the monatomic case, Fig. 1(b).

If a spatial average is taken, Fig. 2(iii), these positions will include both dynamic and static displacements away from the idealised location. The purple line indicates a region containing the same probability of finding an atomic position as shown in (i) and (ii). The change in radius between the red shell (dynamic only) and the purple shell (dynamic and static), is not equal to that of the blue shell (static only) i.e. $\sigma_{u, T}+\sigma_{u, S} \neq \sigma_{u}$. If it is assumed that the two distributions are Gaussian, then the combination of these distributions will also be Gaussian with a standard deviation $\sigma_{u}$ :

$$
\sigma_{u}^{2}=\sigma_{u, T}^{2}+\sigma_{u, S}^{2}
$$

\section{Change in distance - measuring the displacements}

To address the first key question, a measure of the change in distance needs to be established. As discussed previously, for local lattices strain the change in distance is related to the change in the spread of the statistical distribution of the atomic positions. Already within the literature there are two common measures of spread used:

\footnotetext{
${ }^{2}$ this is the same expression as eq.10 in [25], but a different nomenclature here has been used for clarity
}

a) Standard deviation - calculated as:

$$
\sigma=\sqrt{\frac{1}{n} \sum_{n}\left(x_{n}-\mu\right)^{2}}
$$

b) Mean absolute deviation - calculated as:

$$
\xi=\frac{1}{n} \sum_{n}\left|x_{n}-\mu\right|
$$

where $x$ are data values for $n$ data points, and $\mu=\frac{1}{n} \sum_{n} x_{n}$. For Gaussian distributions (e.g. isotropic displacements) the two descriptors may be related to one another, $\xi=\sqrt{\frac{2}{\pi}} \sigma$. Therefore, whilst either statistical method could theoretically be employed, it is suggested that the standard deviation is perhaps more useful.

One of the most commonly used numerical indicators for local lattice strain in the HEA literature is the $\delta$-parameter [26]:

$$
\delta=\sqrt{\sum c_{i}\left(1-\frac{r_{i}}{\bar{r}}\right)^{2}}
$$

where $\bar{r}=\sum c_{i} r_{i}, i$ are atomic species labels, $c$ the concentration and $r$ the radius.

It should be noted that this is of the same form as the definition for static strain $\left(\varepsilon_{u \text {,static }}\right)$, eq. 2 :

$$
\delta=\frac{1}{\bar{r}} \sqrt{\sum c_{i}\left(\bar{r}-r_{i}\right)^{2}}=\frac{\sigma_{r}}{\bar{r}}
$$

Critically, calculating the mean absolute deviation, will not yield a comparable function. Whilst there is a constant of proportionality between the two descriptors for a Gaussian distribution, this relationship will not hold for a nonGaussian distribution such as the distribution of atomic radii being substituted into the sample. This can be particularly significant when predicting strains in multi-component systems, such as HEAs. For example, consider an equiatomic ternary alloy with relative radii $=\{120,126,132\}$, and an equiatomic quaternary alloy with relative radii $=\{120,124$, $128,132\}$. Whilst the average atomic radii, $\bar{r}$, for both alloys would be 126 , these materials would be expected to exhibit different levels of local lattice strain. However, whilst $\sigma_{\text {tern }}=4.90$ and $\sigma_{\text {quat }}=4.47$, both have a value $\xi=4$, suggesting that the mean absolute deviation, $\xi$, may not be suitable for calculating strain.

Proceeding with the standard deviation, there are two further points to consider about the distribution being measured. First, the directionality in which the displacements are being determined. A displacement in 3D will be described in cartesian coordinate space by $\left(x_{n}, y_{n}, z_{n}\right)$. However, when describing a displacement away from a site, it could be a displacement in a given direction, or the absolute displacement given by $R=\sqrt{x^{2}+y^{2}+z^{2}}$. Most commonly, experimental methods will yield the displacement in 
a given direction, rather than the absolute displacement, and therefore this method is suggest for more general adoption.

Second, the way in which the change in distance is being described. Rather than displacements away from the idealised sites, $u$, considered thus far, an entirely equivalent description of these distortions can be made in term of changes in the bond lengths, $l$ :

$$
\varepsilon_{l, \text { static }}=\frac{\sigma_{l, S}}{\mu_{l}}
$$

As previously, both thermal and static effects will contribute to the distribution in bond lengths, and it follows that $\sigma_{l}^{2}=$ $\sigma_{l, T}^{2}+\sigma_{l, S}^{2}$.

For the idealised hard sphere case, it is possible to relate the distribution in $u$ to that in $l$, as $\mu_{l}=2 \bar{r}$ and $\sigma_{l}^{2}=\sigma_{u}^{2}+\sigma_{u}^{2}$ It would follow that $\varepsilon_{u}=\sqrt{2} \varepsilon_{l}$. However, it is found that this will be an upper bound as it does not account for the affect that bonding has on the system.

Thus far, atoms have been described as oscillating hard spheres, which, whilst a good approximation, is limited when used to describe real structures. For example a hard sphere model used to describe a substitutional solid solution would require all atoms in a structure to be displaced away from their idealised sites, irrespective of their distance from the substitution location. In reality, bonds between atoms will store some energy from the distortion, acting like springs. Consequently, whilst the nearest neighbours will experience the greatest displacement, the next nearest neighbours will experience a lesser effect etc. A similar argument can be made for the thermal oscillations. Thermal movement of an atom will result in the motion of the neighbouring atoms due to the bonds connecting them, and is commonly observed in total scattering data, Fig. 3.

Non-bonded atoms move independently, and so the distributions of interatomic distances for different coordination shells have the same $\sigma$. Consequently, all peaks in the corresponding pair distribution function (PDF) (black) have the same width [27]. In contrast, rigidly bonded atoms, connected with 'solid rods', will be constantly held at a fixed distance from one another, and so all PDF peaks are deltafunctions (grey). More realistically, for bonded atoms there will be some correlation between the motion of the nearest neighbouring atoms, whilst atoms in higher coordination shells will move independently. Consequently, the widths of the nearest neighbour peaks will be narrower than the nonbonding hard sphere case (red).

This effect of correlated motion is important, both in terms of the experimental method used and the quantification. Fig. 4 shows the strain calculated from the spring model large box simulations from Owen et al. [25], where atoms have an equal scattering length and a 5\% difference in atomic radius.

The strain has been computed using three quantifications: $\varepsilon_{u}$ calculated using eqs. 2 and 3 (closed circles), $\varepsilon_{l}$ calculated using eq. 8 (open circles), and using a third method
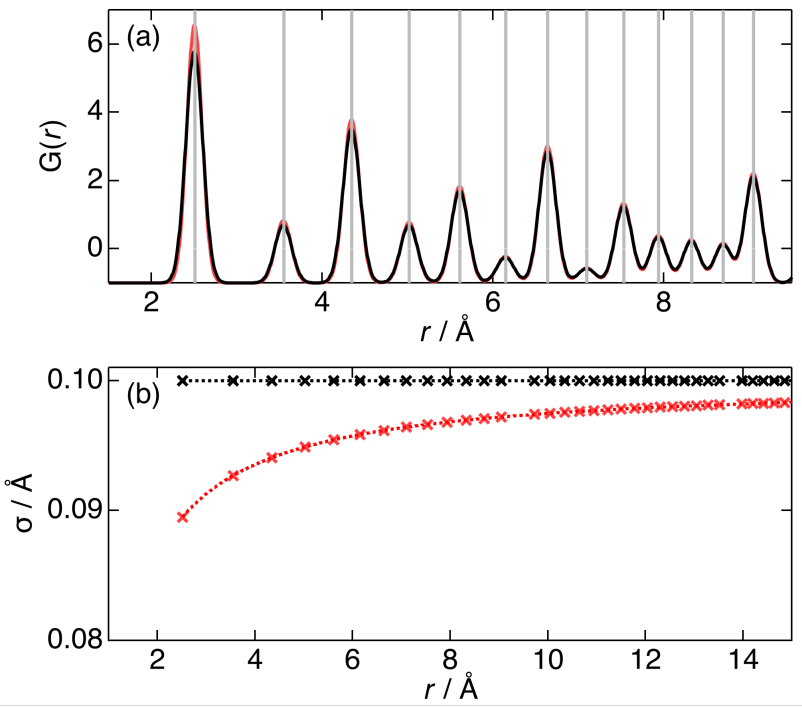

Figure 3: (a) Calculated normalised pair distribution functions (PDFs) plotted; grey - hard rods, black - independent Gaussians, red - correlated motion. (b) Standard deviations of the peaks calculated for the same models as in (a). The grey hard models is not shown as the width is equal to zero for all peaks.

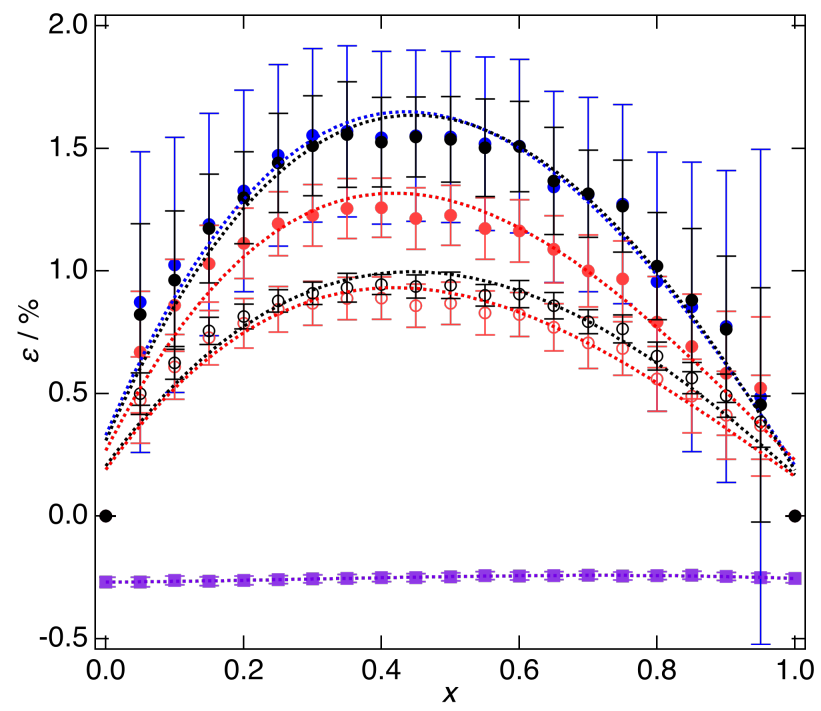

Figure 4: Different methods of calculating strain from large box spring models from [25]. Closed circles $-\varepsilon_{u}$ values, Open circles $-\varepsilon_{l}$ values, Squares - peak position calculation. Black calculated from the large box model directly, Red - calculated from fitting single PDF peak fits, Blue - from PDFGui fitting of whole PDF, Purple - using peak positions calculated from fitting individual peaks, and calculated values from PDFGui whole PDF fitting.

sometimes quoted in the HEA literature:

$$
\varepsilon_{\mathrm{pos}}=\frac{\mu_{l, \mathrm{obs}}-\mu_{l, \mathrm{calc}}}{\mu_{l, \mathrm{calc}}}
$$

[19] (squares) where $\mu_{l}$ is the average interatomic distance (i.e. bond length for first nearest neighbours). Several different data sources have been used: the large box model (black), 
fitting the first PDF peak (red), fitting the whole PDF (blue). In addition, the $\varepsilon_{\text {pos }}$ calculation uses the PDF peak positions in conjunction with calculated values from the whole PDF fit (purple).

Considering the $\varepsilon_{u}$ values (closed circles), the box calculation (black) and whole PDF fitting (blue) are in good agreement, whilst the first peak fitting (red) is substantially different. The first two methods calculate the strain as if the atoms are independent; in contrast, the first peak (red) will be affected by correlated motion and, therefore, has an apparently lower $\sigma_{u}$ and consequently $\varepsilon_{u}$.

Comparing the $\varepsilon_{l}$ values (open circles), the two methods (red/black) are found to be in in good agreement. Unlike the $\varepsilon_{u}$ case, both methods will capture correlated motion. The slight difference between these two data sets, is likely due to Gaussian fitting not precisely capturing the asymmetry of the peaks that arise from the Morse potential. These values are lower than the $\varepsilon_{u}$ values by $\sim \sqrt{2}$, as discussed above.

Finally, it can be seen that the calculation $\varepsilon_{\text {pos }}$ does not capture the local strain in the material. There is no apparent change in $\varepsilon_{\text {pos }}$ with concentration (as would be expected). Further, the value appears to be negative, even for the monatomic species. This is most likely a consequence of the peak asymmetry caused by the Morse potential. With experimentally measured data an additional problem would be an apparent difference in lattice parameter across the $r$-range in a PDF due to instrumental effects (see [28]).

Importantly, both $\varepsilon_{u}$ and $\varepsilon_{l}$ will capture the local strain in the lattice, with the difference being the effect of correlated motion. Which quantification should be used will be dependent on the measurement technique or the intended use of the strain calculation e.g. relating it to a bond dependent physical property.

\section{Reference state}

With respect to the second key question, all of the formulations for strain require a reference state (e.g. $\bar{r}, \mu_{l}$ etc.). Thus, the $\varepsilon$ values calculated can vary substantially depending on the reference chosen. For example, $\bar{r}$ could be calculated theoretically, although a number of methods exist, or determined from experiment (e.g. from a lattice parameter using hard-sphere assumptions). Either way, it is essential that the reference state is clearly defined.

Ideally the reference state should be determined using the same method as the $\sigma$ values e.g. if the $\sigma_{u}$ is determined from Bragg data, then these data should also be used to calculate $\bar{r}$. If an alternative reference state is used then it must be defined explicity and justified appropriately.

\section{Data collection methods}

There are a number of different experimental techniques and predictive methodologies that could be used to assess local lattice strain. Each of these provide relevant information that will be more or less suited to the use of either $\varepsilon_{u}$ and $\varepsilon_{l}$. As such, it is important for those working in the field to be aware of the type of data available from these methods, and the quantification that can describe it. Since a full discussion of the suitability of these techniques, their accuracy and issues associated with them for the quantification of local lattice strain has been made elsewhere [5], this section will focus on relating the methods to the quantifications described thus far.

\subsection{X-ray and Neutron scattering - Bragg data}

This is the most common experimental method used to probe atomic structure. Importantly, data of this type represents scattering from all atoms in the material (i.e. spatially averaged) over a period of time (i.e. temporally averaged). Full pattern fitting, can yield the lattice parameter of the material from which $\bar{r}$, can be calculated, from knowledge of the structure and assuming a hard sphere model.

If the data are of higher quality, then it may be possible to obtain additional information from the decay in the Bragg peaks. Local displacements in the structure can be modelled by a decaying form factor using a strain modified DebyeWaller factor $\left(B_{T, S}\right)$ :

$$
B_{T, S}=B_{T}+B_{S}=8 \pi^{2}\left[\sigma_{u, T}^{2}+\sigma_{u, S}^{2}\right]
$$

where $B_{T}$ and $B_{S}$ are the thermal and static Debye-Waller factors respectively.

Importantly, the offsite displacements measured in a diffraction experiment correspond to the projection of the displacements onto the scattering vector. If, as is the case for most metals, the material is isotropic then all the directions are identical, $\left\langle u^{2}\right\rangle_{t, s}=\sigma^{2}=\sigma_{x}^{2}=\sigma_{y}^{2}=\sigma_{z}^{2}$.

This method is highly reliant on the accuracy of fit to the data, and can only be done successfully if all factors relating to the peak intensity have been accounted for, e.g. absorption, fluorescence and texture.

One common source of confusion in the literature can arise from the use of the expression:

$$
\varepsilon_{h k l, I I}=\frac{d_{h k l}-d_{h k l, 0}}{d_{h k l, 0}}
$$

where $d_{h k l}$ is the measured peak spacing and $d_{h k l, 0}$ a theoretical $d$-spacing. This is a strain, but is the strain on a specific family of planes, which is known as a Type II strain [23].

Similarly, attempts have been made to estimate strain from Bragg peak width, using metrics such as:

$$
\varepsilon_{h k l, I I I}=\frac{\sigma_{h k l}-\sigma_{h k l, 0}}{d_{h k l, 0}}
$$

where $\sigma_{h k l}$ is the width of an individual Bragg peak, and $\sigma_{h k l, 0}$ the theoretical width (from e.g. instrumental broadening, size effects etc.). Again, whilst this is a measure of strain, these are Type III strains [23].

\subsection{X-ray and Neutron scattering - Total scattering}

An alternative method for extracting lattice strain information is from total-scattering data, and more specifically 
pair distribution function (PDF) data (see [25]). Again this method both spatially and temporally averages. From an individual peak in the PDF it is possible to obtain $\mu_{l}$ and $\sigma_{l}$, and so measure a value of $\varepsilon_{l, \text { static }}$. Additionally, by fitting across the entire $r$-range, it is possible to calculate $\sigma_{u \text {,static }}$. Such fitting will also yield a lattice parameter, from which $\bar{r}$ can be calculated, making it simultaneously possible to obtain $\varepsilon_{u \text {,static }}$.

As discussed previously, there is some quantification in the literature using $\varepsilon_{\text {pos }}$; however, as outlined above, this fails to capture the desired information.

\subsection{EXAFS}

This method is similar to total scattering in that it records interatomic displacements and so can yield $\varepsilon_{l \text {,static }}$ values. Unlike total scattering it allows the direct probing of the distributions around individual atoms, potentially providing species specific strains. However, the accessible $r$-range measured with EXAFS is much shorter than total scattering, so no comparative $\varepsilon_{u \text {,static }}$ value can be easily measured.

To allow the species specific strains to be compared with other experimental measures, the patterns from all the elements would need to be measured and averaged. Alternatively, the individual atomic contributions could be compared with those calculated from theoretical models or partial pair distribution functions extracted from large box modelling of total scattering data. As previously, this method both temporally and spatially averages.

\subsection{Transmission Electron Microscopy}

Transmission electron microscopy is capable of yielding high resolution images of atomic columns in a material. From such an image it should be possible to measure an atomic size, which would incorporate the thermal and static offsite displacements $u$. However, what proportion of this radius can be attributed to the size of the electron cloud, the thermal displacements and static displacements (averaged down the column of atoms) is unclear. It may, however, still be possible to estimate the interatomic distances $l$. In either case though it will always be difficult to estimate the temperature of the sample, particularly under the heating effect of the electron beam. Further, it is necessary to assume that the thinning of the sample will not effect the local strains in the system. Finally the quality of such data are highly dependent on the resolution of the instrument.

\section{5. $A b$ initio methods}

Using $A b$ initio methods it is possible to obtain a prediction of the lattice strain from an atomic model. Such models are static, but can be spatially averaged to give bond length distributions and the associated statistical parameters. This allows the prediction of a $\varepsilon_{l \text {,static,theo }}$ which can be compared with experimentally determined measures.

It is also possible to use this method to calculate offsite displacement parameters and so $\varepsilon_{u \text {,static }}$. However, this requires the determination of an 'idealised atomic position'. This may require minimisation of $\sigma_{u}$ to work out where the idealised sites are located, providing a potential source of error.

One advantage of such a method is that it allows for relaxation fo the structure, i.e. expansion of the lattice under increased strain. However, such simulations are performed at $0 \mathrm{~K}$, and whilst extrapolations are possible to model higher temperatures, the accuracy of such calculations can be highly system dependent.

\section{Concluding remarks}

At present there is no commonly accepted method for quantifying local lattice strain, without which it is not possible to compare data from different alloys obtained using different methods. If the validity of the highly-strained lattice hypothesis is to be established, systematic quantification of local strains in host of alloys of increasing compositional complexity is required. The assessment and quantification of such strains requires two key pieces of information: a change in distance, and a comparative reference state. Local displacements, arising from dynamic thermal motion as well as static alloying effects, will combine to alter the widths of the statistical distributions of the positions of the atomic centres.

Parameters for describing such the widths of such distributions include standard deviations and mean absolute deviation. However, here we suggest that a standard deviation $(\sigma)$ is a more useful measure for comparison to predicted $\delta$ values, which are commonly used by the HEA community as an indicator for local strain. To extract information about the static displacements, the system will need to be spatially averaged and the effect of the dynamic displacements removed. As such, variable temperature measurements need to be made on these systems, to allow the separation of these two effects.

Additionally, it has been discussed how the distribution in the bond lengths, $l$, can also be used to assess the strain, in an analogous manner. Importantly, such a measure will incorporate variations in the bond length due to the type of bonding and the correlated motion in the system. As such, this method may give a measure that is more useful when describing physical properties that are bond dependent.

Methodologies appropriate for measuring or predicting local lattice strains have been discussed, with specific reference to whether they describe $u$ or $l$-type distributions. The majority of experimental methods used to probe local lattice strain yield distributions in $l$ rather than in $u$. In addition, calculations of the distributions from ab initio methods in $l$ will be simpler, and with lower error, than those performed in $u$. Consequently, for practical application and cross comparison, it is thought that expressing the distributions in terms of $l$ may be the more useful of the two quantifications.

Given the statistical nature of the displacement, the most logical reference state is the corresponding average ( $\bar{r}$ for $u$, $\mu_{l}$ for $l$ ) which should ideally be obtained from the same data set as the distribution width.

The use of a common mathematical quantification, such 
as that suggested here, should enable meaningful comparisons to be made between results across a range of alloys obtained using different experimental techniques and theoretical predictions. Such comparisons will be of great benefit to the field moving forwards and in determining the significance of a given level of local lattice strain in a particular system. By making systematic measurements of local lattice strain in a host of materials, from binary alloys to compositionally complex materials, under variable temperature conditions, and using a common quantification, it may be possible to establish the effect of these distortions on the physical properties of a material, and whether indeed the highentropy alloys can be considered highly-strained.

\section{Acknowledgements}

L. R. Owen would like to thank Gonville and Caius College for their support.

\section{References}

[1] J.-W. Yeh, S.-K. Chen, S.-J. Lin, J.-Y. Gan, T.-S. Chin, T.-T. Shun, C.-H. Tsau, S.-Y. Chang, Nanostructured high-entropy alloys with multiple principal elements: Novel alloy design concepts and outcomes, Advanced Engineering Materials 6 (5) (2004) 299-303. doi : 10.1002/adem. 200300567.

[2] J. Yeh, Recent progress in high-entropy alloys, Annales de chimie Science des Materiaux 31 (2006) 633-648.

[3] E. Pickering, N. Jones, High entropy alloys: A critical assessment of their founding principles and future prospects, International materials reviews 61 (3) (2016) 183-202.

[4] D. Miracle, O. Senkov, A critical review of high entropy alloys and related concepts, Acta Materialia 122 (2017) 448 - 511.

[5] L. R. Owen, N. G. Jones, Lattice distortions in high-entropy alloys, Journal of Materials Research 33 (19) (2018) 2954-2969. doi:10. 1557/jmr. 2018.322.

[6] F. Wang, Y. Zhang, G. Chen, Atomic packing efficiency and phase transition in a high entropy alloy, Journal of Alloys and Compounds 478 (2009) 321-324.

[7] B. Murty, J.-W. Yeh, S. Ranganathan, High-Entropy Alloys, Elsevier, 2014.

[8] Y. Zuo, S. Maiti, W. Steurer, R. Spolenak, Size-dependant plasticity in an $\mathrm{Nb}_{25} \mathrm{Mo}_{25} \mathrm{Ta}_{25} \mathrm{~W}_{25}$ refractory high-entropy alloy, Acta Materialia 65 (2014) 85-97.

[9] O. Senkov, J. Scott, S. Senkova, D. Miracle, C. Woodward, Microstructure and room temperature properties of a high-entropy TaNbHfZrTi alloy, Journal of Alloys and Compounds 509 (20) (2011) $6043-6048$.

[10] Z. Wu, H. Bei, G. Pharr, E. George, Temperature dependence of the mechanical properties of equiatomic solid solution alloys with facecentered cubic crystal structures, Acta Materialia 81 (2014) 428 441.

[11] I. Toda-Caraballo, P. E. R.-D. del Castillo, Modelling solid solution hardening in high entropy alloys, Acta Materialia 85 (2015) $14-23$.

[12] C. R. LaRosa, M. Shih, C. Varvenne, M. Ghazisaeidi, Solid solution strengthening theories of high-entropy alloys, Materials Characterization 151 (2019) $310-317$.

[13] J. Yeh, S. Chang, Y. Hong, S. Chen, S. Lin, Anomalous decrease in $\mathrm{X}$-ray diffraction intensities of $\mathrm{Cu}-\mathrm{Ni}-\mathrm{Al}-\mathrm{Co}-\mathrm{Cr}-\mathrm{Fe}-\mathrm{Si}$ alloy systems with multi-principal elements, Materials Chemistry and Physics 103 (2007) 41-46.

[14] J. Yeh, Physical metallurgy of high-entropy alloys, Journal of materials 67 (2015) 2254-2261.

[15] N. L. Okamoto, K. Yuge, K. Tanaka, H. Inui, E. P. George, Atomic displacement in the CrMnFeCoNi high-entropy alloy - A scaling factor to predict solid solution strengthening, AIP Advances 6 (12) (2016) 125008

[16] W. Guo, W. Dmowski, J. Noh, P. Rack, P. Liaw, T. Egami, Local atomic structure of a high-entropy alloy: An X-ray and neutron scattering study, Metallurgical and Materials Transactions A 44 (2012) 1994-1997.

[17] L. Santodonato, Y. Zhang, M. Feygenson, C. Parish, M. Gao, R. Weber, J. Neuefeind, Z. Tang, P. Liaw, Deviation from high-entropy configurations in the atomic distributions of a multi-principal-element alloy, Nature Communications 6 (5964).

[18] L. Owen, E. Pickering, H. Playford, H. Stone, M. Tucker, N. Jones, An assessment of the lattice strain in the crmnfeconi high-entropy alloy, Acta Materialia 122 (2017) 11-18.

[19] Y. Tong, S. Zhao, H. Bei, T. Egami, Y. Zhang, F. Zhang, Anomalous local distortion in bec refractory high-entropy alloys, https://arxiv.org/abs/1902.09279.

[20] H. Oh, D. Ma, G. Leyson, B. Grabowski, E. Park, F. Körmann, D. Raabe, Lattice distortions in the feconicrmn high entropy alloy studied by theory and experiment, Entropy 18 (2016) 321.

[21] S. S. Sohn, A. Kwiatkowski da Silva, Y. Ikeda, F. Körmann, W. Lu, W. S. Choi, B. Gault, D. Ponge, J. Neugebauer, D. Raabe, Ultrastrong Medium-Entropy Single-Phase Alloys Designed via Severe Lattice Distortion, Advanced Materials 31 (8) (2018) 1807142.

[22] S. Mu, S. Wimmer, S. Mankovsky, H. Ebert, G. M. Stocks, Influence of local lattice distortions on electrical transport of refractory high entropy alloys, Scripta Materialia 170 (2019) 189-194.

[23] P. Withers, H. Bhadeshia, Residual stress. part 1 - measurement techniques, Materials Science and Technology 17 (4) (2001) 355-365.

[24] Y. Zhang, T. T. Zuo, Z. Tang, M. C. Gao, K. A. Dahmen, P. K. Liaw, Z. P. Lu, Microstructures and properties of high-entropy alloys, Progress in Materials Science 61 (2014) 1 - 93.

[25] L. Owen, H. Stone, H. Playford, The assessment of local lattice strains in alloys using total scattering, Acta Materialia 170 (2019) 38-49.

[26] S. Fang, X. Xiao, L. Xia, W. Li, Y. Dong, Relationship between the widths of supercooled liquid regions and bond parameters of $\mathrm{Mg}$ based bulk metallic glasses, Journal of Non-Crystalline Solids 321 (1) (2003) $120-125$.

[27] T. Egami, S. Billinge, Underneath the Bragg Peaks, Pergamon Materials Series, Elsevier, Oxford, UK, 2012.

[28] X. Qiu, E. S. Božin, P. Juhas, T. Proffen, S. J. L. Billinge, Reciprocalspace instrumental effects on the real-space neutron atomic pair distribution function, Journal of Applied Crystallography 37 (1) (2004) $110-116$. 